\title{
GPU Acceleration of Bounded Model Checking with ParaFROST
}

\author{
Muhammad Osama ${ }^{(\bowtie)}(\mathbb{D}$ and Anton Wijs (D \\ Eindhoven University of Technology, \\ Eindhoven, The Netherlands \\ \{o.m.m.muhammad,a.j.wijs\}@tue.nl
}

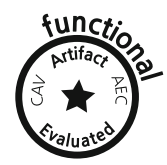

\begin{abstract}
The effective parallelisation of Bounded Model Checking is challenging, due to SAT and SMT solving being hard to parallelise. We present PARAFROST, which is the first tool to employ a graphics processor to accelerate BMC, in particular the simplification of SAT formulas before and repeatedly during the solving, known as pre- and inprocessing. The solving itself is performed by a single CPU thread. We explain the design of the tool, the data structures, and the memory management, the latter having been particularly designed to handle SAT formulas typically generated for BMC, i.e., that are large, with many redundant variables. Furthermore, the solver can make multiple decisions simultaneously. We discuss experimental results, having applied PARAFROST on programs from the Core C99 package of Amazon Web Services.
\end{abstract}

Keywords: Bounded model checking $\cdot$ SAT solving $\cdot$ GPU computing

\section{Introduction}

Bounded Model Checking (BMC) [5] determines whether a model $M$ satisfies a certain property $\varphi$ expressed in temporal logic, by translating the model checking problem to a propositional satisfiability (SAT) problem or a Satisfiability Modulo Theories (SMT) problem. The term bounded refers to the fact that the BMC procedure searches for a counterexample to the property, i.e., an execution trace, which is bounded in length by an integer $k$. If no counterexample up to this length exists, $k$ can be increased and BMC can be applied again. This process can continue until a counterexample has been found, a user-defined threshold has been reached, or it can be concluded (via $k$-induction [38]) that increasing $k$ further will not result in finding a counterexample. CBMC [14] is an example of a successful BMC model checker that uses SAT solving. CBMC can check ANSI-C programs. The verification is performed by unwinding the loops in the program under verification a finite number of times, and checking whether the bounded

M. Osama-This work is part of the GEARS project with project number TOP2.16.044, which is (partly) financed by the Netherlands Organisation for Scientific Research (NWO).

(C) The Author(s) 2021

A. Silva and K. R. M. Leino (Eds.): CAV 2021, LNCS 12760, pp. 447-460, 2021.

https://doi.org/10.1007/978-3-030-81688-9_21 




(a) The amount of variable redundancy in CBMC formulas

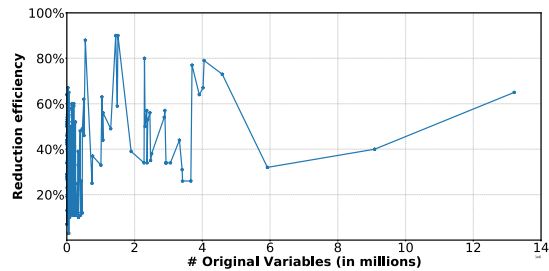

(b) The amount of variable redundancy w.r.t. the number of variables

Fig. 1. Variable redundancy in CBMC SAT formulas

executions of the program satisfy a particular safety property [22]. These properties may address common program errors, such as null-pointer exceptions and array out-of-bound accesses, and user-provided assertions.

The performance of BMC heavily relies on the performance of the solver. Over the last decade, efficient SAT solvers $[3,6,17,26]$ have been developed and applied for BMC [5,10-12,25]. Effectively parallelising BMC is hard. Parallel SAT solving often involves running several solvers, each solving the problem in its own way [18]. For BMC, multiple solvers can be used to solve the problem for different values of the bound $k$ in parallel $[1,21]$. However, in these approaches, the individual solvers are still single-threaded.

Recently, Leiserson et al. [23] concluded that in the future, advances in computational performance will come from many-threaded algorithms that can employ hardware with a massive number of processors. Graphics processors (GPUs) are an example of such hardware. Multi-threaded BMC model checkers have been proposed, such as in $[13,19,35]$, but these address tens of threads, not thousands.

In this paper, we propose the application of GPUs to accelerate SAT-based BMC. To the best of our knowledge, this is the first time this is being addressed. Recently, GPUs have been applied for explicit-state model checking and graph analysis $[8,9,40,41]$. In SAT solving, we used GPUs to accelerate test pattern generation [31], metaheuristic search [42], preprocessing [32,33] and inprocessing [34]. In these operations, a given SAT formula is simplified, i.e., it is rewritten to a formula with fewer variables and/or clauses, while preserving satisfiability, using various simplification rules. In preprocessing, this is only done once before the solving starts, while in inprocessing, this is done periodically during the solving. While the impact of accelerating these procedures has been demonstrated [34], its impact on BMC has not yet been addressed.

The structure of typical BMC SAT formulas suggests that GPU pre- and inprocessing will be effective. Figure 1a shows for a BMC benchmark set taken from the Core C99 package of Amazon Web Services (AWS) ${ }^{1}$ [2], consisting of 168 problems of various data structures, that propositional formulas produced by CBMC tend to have a substantial amount of redundant variables that can

\footnotetext{
${ }^{1}$ We thank Daniel Kroening and Natasha Jebbo for pointing us to this package.
} 
be removed using simplification procedures. For approximately $50 \%$ of the cases, $40 \%$ of the variables can be removed. Furthermore, Fig. 1b presents the amount of redundancy in relation to the total number of variables in the formula. It indicates that when a formula contains one million variables or more, at least $25 \%$ of those are redundant, and often many more. In the benchmark set, the maximum number of variables in one formula is 13 million (encoding the verification of the priority-queue shift-down routine), of which $65 \%$ is redundant. In contrast, the largest formula we encountered in the application track of the 2013-2020 SAT competitions that is not encoding a verification problem only has 0.2 million variables (it encodes a graph coloring problem [29]).

Contributions. We present the SAT solver PARAFROST that applies Conflict Driven Clause Learning (CDCL) [26] with GPU acceleration of pre- and inprocessing [32-34], tuned for BMC. It has been implemented in CUDA C++ v11 [28], is based on CADICAL [6], and interfaces with CBMC.

Having to deal on a GPU with large formulas with a lot of redundancy offers particular challenges. The elimination of variables typically leads to actually adding new clauses, and since the amount of memory on a GPU is limited, this cannot be done carelessly. Therefore, first of all, we have worked on compacting the data structure used to store formula clauses in PARAFROST as much as possible, while still allowing for the application of effective solving optimisations. Second of all, we introduce memory-aware variable elimination, to avoid running out of memory due to adding too many new clauses. In practice, we experienced this problem when applying the original procedure of [34] for BMC.

Additionally, to support BMC, PARAFROST must be an incremental solver, i.e., it must exploit that a number of very similar SAT problems are solved in sequence [16]. The procedure in [34] does not support this, so we extended it.

Finally, because of the many variables in BMC SAT formulas, PARAFROST supports Multiple Decision Making (MDM) in the solving procedure, as presented in [30]. With MDM, multiple decisions can be made at once, periodically during the solving. In case there are many variables, there is more potential to make many decisions simultaneously. We have generalised the original MDM decision procedure [30], making it easier to integrate MDM in solvers other than MiniSat and Glucose [3]. The effectiveness of MDM in BMC has never been investigated before, nor has been combined with GPU pre- and inprocessing.

\section{Background}

SAT Solving. We assume that SAT formulas are in conjunctive normal form (CNF). A CNF formula is a conjunction of $m$ clauses $C_{1} \wedge \cdots \wedge C_{m}$, and each clause $C_{i}$ is a disjunction of $n$ literals $\ell_{1} \vee \cdots \vee \ell_{n}$. A literal is a Boolean variable $x$ or its negation $\neg x$, also referred to as $\bar{x}$. The domain of all literals is $\mathbb{L}$. A clause can be interpreted as a set of literals, i.e., $\left\{\ell_{1}, \ldots, \ell_{n}\right\}$ encodes $\ell_{1} \vee \ldots \vee \ell_{n}$, and a SAT formula $\mathcal{S}$ as a set of clauses, i.e., $\left\{C_{1}, \ldots, C_{m}\right\}$ encodes $C_{1} \wedge \ldots \wedge C_{m}$. With $\operatorname{Var}(C)$, we refer to the set of variables in $C: \operatorname{Var}(C)=\{x \mid x \in C \vee \bar{x} \in C\}$. The set $\mathcal{S}_{\ell}$ consists of all clauses in $\mathcal{S}$ containing $\ell: \mathcal{S}_{\ell}=\{C \in \mathcal{S} \mid \ell \in C\}$. 
In CDCL, clauses are LEARNT or ORIGINAL. A LEARNT clause has been derived by the CDCL clause learning process during solving, and an ORIGINAL clause is part of the formula. We refer with $\mathcal{L}$ to the set of LEARNT clauses.

For a set of assignments $\Sigma$, consisting of all literals that have been assigned true, a formula $\mathcal{S}$ evaluates to true iff $\forall C \in \mathcal{S} . \exists \ell \in C . \ell \in \Sigma$. When a decision is made, a literal is picked and added to $\Sigma$. Each assignment is associated with a decision level (time stamp) to monitor the assignment order. We call a clause $C$ unit iff a single literal in it is still unassigned, and the others are assigned false, i.e., $|\operatorname{Var}(C) \backslash \operatorname{Var}(\Sigma)|=1$ and $C \cap \Sigma=\emptyset$.

Variable-Clause Elimination (VCE). Variables and clauses can be removed from formulas by applying simplification rules $[15,20]$. They rewrite a formula to an equi-satisfiable one with fewer variables and/or clauses. Applying them is referred to as pre- and inprocessing, before and during the solving, respectively.

Incremental Bounded Model Checking. Since 2001, incremental BMC has been applied to hardware and software verification $[16,39]$. It relies on incremental SAT solving [16]. In CDCL, clauses are learnt during the solving each time a wrong decision has been made, to avoid making those decisions again in the future. Incremental SAT solving builds on this: when multiple SAT formulas with similar characteristics are solved sequentially, then in each iteration, the clauses learnt in previous iterations are reused. An efficient approach to add and remove clauses is by using assumptions [16], which are initial assignments.

For BMC, the transition relation of a system design and the (negation of) the property to be verified are encoded in a SAT formula. A predicate $\mathcal{I}\left(s_{0}\right)$ identifies the initial states, $\delta\left(s_{i}, s_{i+1}\right)$ encodes the transition relation at trace depth $i$, and $\mathcal{E}(i)=\bigvee_{0 \leq j \leq i} e\left(s_{j}\right)$ encodes the reachability of an error state up to trace depth $i$, where $e\left(s_{j}\right)$ is true iff state $s_{j}$ is an error state. For incremental BMC, additional unit clauses $\sigma_{i}$ are used. These predicates are combined to define the following series of SAT formulas $\mathcal{S}(i)$ that must be solved incrementally:

$$
\begin{aligned}
\mathcal{S}(0) & =\mathcal{I}\left(s_{0}\right) \wedge\left(\mathcal{E}(0) \vee \sigma_{0}\right), \text { under assumption } \neg \sigma_{0} \\
\mathcal{S}(i+1) & =\mathcal{S}(i) \wedge \delta\left(s_{i}, s_{i+1}\right) \wedge \sigma_{i} \wedge\left(\mathcal{E}(i+1) \vee \sigma_{i+1}\right), \text { under assumption } \neg \sigma_{i+1}
\end{aligned}
$$

Formula $\mathcal{S}(i)$ is satisfiable iff an error state is reachable via a trace with a length up to $i[16,39]$. At iteration $i+1$, we know that $\mathcal{E}(i)$, included via $\mathcal{S}(i)$, cannot be satisfied (otherwise iteration $i+1$ would not have been started). This means that $\mathcal{E}(i)$ must be removed to avoid that $\mathcal{S}(i+1)$ is unsatisfiable. To effectively remove $\mathcal{E}(i), \sigma_{i}$ is assigned true, resulting in $\mathcal{E}(i) \vee \sigma_{i}$ being satisfied. In general, at iteration $i, \sigma_{i}$ is assigned false, while in iterations $i^{\prime}>i$, it is assigned true.

GPU Programming. CUDA [28] is NVIDIA's parallel computing platform that can be used to develop general purpose GPU programs. A GPU consists of multiple streaming multiprocessors (SMs), and each SM contains several streaming processors (SPs). A GPU program consists of a host part, executed on a $\mathrm{CPU}$, and device functions, or kernels, executed on a GPU. Each time a kernel is launched, the number of threads that need to execute it is given. On the SPs, the threads are executed. Compared to a CPU thread, GPU threads perform 


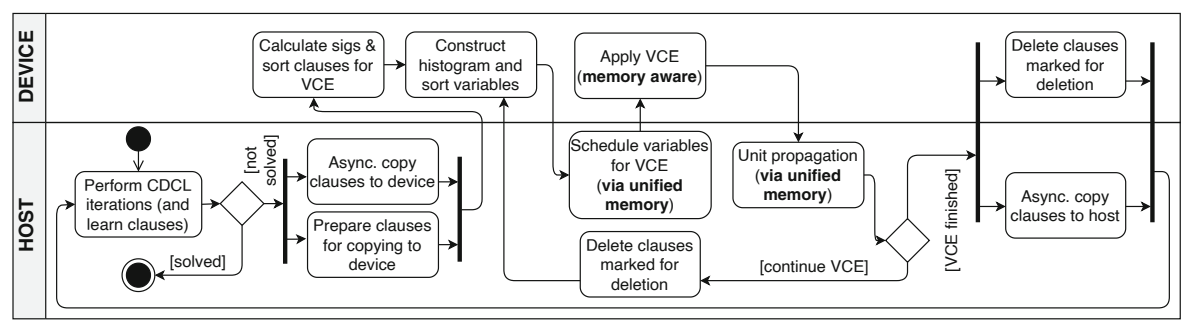

Fig. 2. An activity diagram for the workflow of PARAFROST.

a relatively simple task. In particular, they read some data, perform a computation, and write the result. This allows the SPs to switch contexts easily. In practice, one to two orders of magnitude more threads are typically launched than the number of SPs, which results in hiding the memory latency: whenever a thread is waiting for some data, the associated SP can switch to another thread.

A GPU has various types of memory. Relevant here are registers and global memory. Global memory is used to copy data between the host and the device. Registers are used for on-chip storage of thread-local data. Global memory has a much higher latency than registers. We use unified memory [28] to store clauses. Unified memory creates one virtual memory pool for host and device. In this way, the same memory addresses can be used by the host and the device, combining the main memory of the host side and the global memory of the device side.

\section{GPU-Accelerated Bounded Model Checking}

We implemented PARAFROST ${ }^{2}$ with CUDA C++ v11. It is a hybrid CPU-GPU tool, with (sequential) solving done on the host side, and (parallel) VCE done on the device side. An interface with CBMC is implemented in $\mathrm{C}++$. CBMC is patched to read a configuration file before PARAFROST is instantiated. This file contains all options supported by PARAFROST.

The Workflow. Figure 2 presents the general workflow of PARAFROST in the form of an activity diagram with host and device lanes. The diagram is focused on inprocessing; preprocessing works similarly on the device. First, the host performs a predetermined number of solving iterations. Once those have finished, and (un)satisfiability has not yet been proven, relevant clause data is copied to the global memory. To hide the latency of this operation as much as possible, clauses are copied asynchronously in batches. One batch is copied while the next is formatted for the GPU, as not all clause information on the host side is relevant for the device (see the next paragraph on data structures). On the device, signatures are computed for fast clause comparison, and the clauses are sorted for VCE (more on VCE later). Next, the device constructs a histogram, for fast lookup of clauses, and sorts the variables. The THRUsT library is used

\footnotetext{
2 The tool is available at https://gears.win.tue.nl/software/gpu4bmc.
} 
for sorting. ${ }^{3}$ After that, the host schedules variables for VCE, marking those variables in the global memory using unified memory. Next, the device applies VCE, marking clauses to be removed as DELETED. The host propagates units (literals in unit clauses are assigned true), which directly has an effect on the formula in the global memory. The VCE procedure is repeated until it has been performed a predetermined number of times. After each time, DELETED clauses are removed, and after the last iteration, this is done while the new clauses are copied to the host. Once this has been done, the overall procedure is repeated.

Data Structures and Memory Management. We have worked on making the storage of each clause in the GPU global memory as efficient as possible. However, we also wanted to annotate each clause with sufficient information for effective optimisations. In PARAFROST, the following information is stored for each clause:

- The state field (2 bits) stores if the state is ORIGINAL, LEARNT or DELETED.

- The used field (2 bits) keeps track of how many search iterations a LEARNT clause can still be used. LEARNT clauses are used at most twice [6].

- Two fields (1 bit each) are used for VCE bookmarking.

- The literal block distance (1bd) (26 bits) stores the number of decision levels contributing to a conflict, if there is one [3]. A maximum value of $2^{26}$ turns out to be sufficient. This field is updated when the clause is altered.

- The size (32 bits) of the clause, i.e., the number of literals.

- A signature sig (32 bits) is a clause hash, for fast clause comparison [15].

In addition, a list of literals is stored, each literal taking 32 bits (1 bit to indicate whether it is negated or not, and 31 bits to identify the variable). In total, a clause requires $12+4 t$ bytes, with $t$ the number of literals in the clause. For comparison, MiniSAT only requires $4+4 t$ bytes, but it does not involve the used, lbd and sig fields, thereby not supporting the associated optimisations. CADiCAL [6] uses $28+4 t$ bytes, since it applies solving and VCE on the same structures. In PARAFROST, the GPU is only used for VCE, in which information for probing [24] and vivification [36], for instance, is irrelevant. Finally, in [34], $20+4 t$ bytes are used, storing the same information as PARAFROST.

To store a formula $\mathcal{S}$, a clause array is preallocated in the global memory, and filled with the clauses of $\mathcal{S}$. More space is allocated than the size of $\mathcal{S}$, to allow the addition of clauses that result from VCE. As the amount of allocated space is the limiting factor for the addition of new clauses, we have developed a memory-aware VCE mechanism, which we explain later in the current section.

Parallel VCE. PARAFROST supports the VCE rules substitution (i.e., gate equivalence reasoning), resolution (RES), subsumption elimination (SUB) and eager redundancy elimination (ERE) [15,20]. Substitution applies to patterns representing logical gates, and substitutes the involved variables with their gate definitions. PARAFROST supports AND/OR, Inverter, If Then Else and XOR.

\footnotetext{
${ }^{3}$ https://docs.nvidia.com/cuda/thrust.
} 


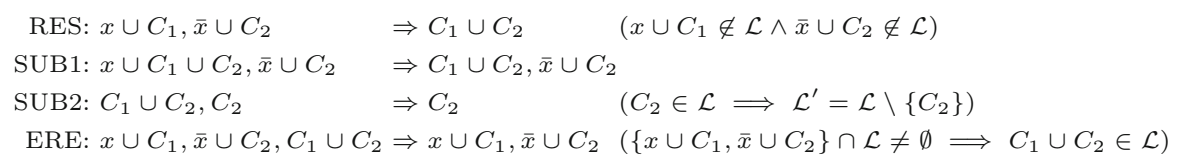

Fig. 3. VCE rules in PARAFROST. $C_{1}$ and $C_{2}$ are non-empty sets of literals.

In Fig. 3, we provide rewrite rules for SUB and RES. If clauses exist in $\mathcal{S}$ of the form expressed by the left hand side of a rule, then the rule is applicable, and the involved clauses are replaced by the clauses (called resolvents) on the right hand side. RES is applicable if there are two clauses of the form $x \cup C_{1}$ and $\bar{x} \cup C_{2}$, and applying it results in replacing those with a clause $C_{1} \cup C_{2}$. SUB consists of two rules; the second is applied once the first is no longer applicable.

Conditions are given between parentheses. For RES, only ORIGINAL clauses are considered. Besides that, if $C_{1} \cup C_{2}$ evaluates to true, it is actually not created. As LEARNT clauses are sometimes deleted during solving, SUB2 should only produce ORIGINAL clauses; if $C_{2}$ is LEARNT before applying the rule, it will become ORIGINAL $\left(\mathcal{L}^{\prime}\right.$ refers to the set of LEARNT clauses after application). For ERE, LEARNT clauses cannot cause the deletion of an ORIGINAL clause.

VCE is applied in parallel by PARAFROST by scheduling sets of mutuallyindependent variables for analysis. Two variables $x$ and $y$ are independent in $\mathcal{S}$ iff $\mathcal{S}$ does not contain a clause containing literals that refer to both variables, i.e., $\mathcal{S}_{x} \cup \mathcal{S}_{\bar{x}}$ and $\mathcal{S}_{y} \cup \mathcal{S}_{\bar{y}}$ are disjoint. This ensures that two threads focussing on $x$ and $y$, respectively, does not lead to data races. In incremental solving, variables referred to by assumptions must be excluded from VCE. In each VCE iteration, a different set $\Psi$ of variables is selected. This is achieved by using an upper-bound $\mu$ for the number of occurrences of a variable in $\mathcal{S}$. After each iteration, $\mu$ is increased, allowing the selection of more variables. PARAFROST supports configuring $\mu$ and the number of VCE iterations.

As already mentioned, clauses that can be removed are marked DELETED before they are removed. The removal of clauses is done once VCE has finished (see Fig. 2) to avoid data races. However, because of this, VCE may at first require more memory to store clauses. The clauses added during VCE must fit in the memory, otherwise the procedure fails. To ensure this, we have developed a memory-aware mechanism for VCE. Next, we explain this mechanism for the RES rule and substitution, as the application of those rules results in new clauses.

Algorithm 1 presents how RES and substitution are applied in PARAFROST. It requires $\mathcal{S}$, stored in a clause array clauses. As clauses are of varying sizes, we need an array references that provides a reference to each clause. In addition, arrays varinfo, cindex and rindex are given, which are filled in the first lines.

At line 1, the kernel VCESCAN is called in which a different thread is assigned to each variable $x \in \Psi$. Each thread checks the applicability of VCE rules on its variable and computes the number of clauses and literals that will be produced by the first applicable rule. A thread with ID $i$ stores the type $\tau$ of the applicable rule (NONE, RESOLVE, or SUBSTITUTE) and the number of clauses $\beta$ and 


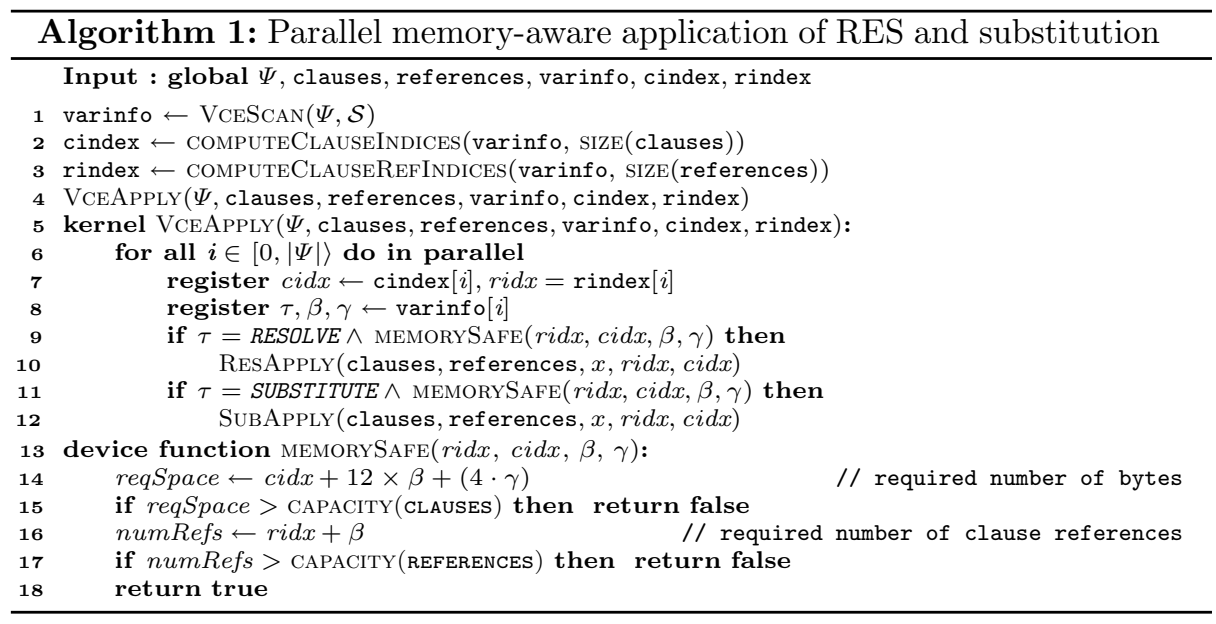

literals $\gamma$ produced by that rule in one integer at varinfo[i]. At lines $2-3$, kernels COMPuteClauseIndices and COMPuteClauseRefIndices are called to add up the $\beta$ 's and $\gamma$ 's to obtain offsets into the arrays references and clauses (the method $\operatorname{sizE}(A)$ refers to the amount of data in array $A$ ). Both methods apply a parallel exclusive prefix sum [37], involving the $\beta$ 's and $\gamma$ 's. The result is that thread $i$, assigned to $x$, is instructed to start writing clause references at references[rindex $[i]]$ and clauses at clauses[cindex $[i]]$ when applying the next VCE rule for $x$. Whether the data actually fits is checked later.

Next, the kernel VCEAPPLY is called (lines 5-12). To each variable in $\Psi$, a thread is assigned. It retrieves the precomputed data (lines 7-8) and either applies the RES rule (lines 9-10), substitution (lines 11-12), or nothing, in case $\tau=$ NONE. However, a condition for applying a rule is that there is enough space, which is checked using the device function MEMORYSAFE (lines 13-18). The amount of allocated space for $A$ is reflected by CAPACITY (A), and MEMORYSAFE checks if there is enough space in clauses, starting at cidx (lines 14-15). If there is, it is checked if the references can be stored in references (lines 16-17).

\section{Multiple Decision Making in Incremental Solving}

Given the fact that BMC SAT formulas often have many variables, a recently proposed extension of CDCL [30], in which periodically multiple decisions are made (MDM) at the same time, has much potential to speed up BMC. When the MDM method is called, it constructs a set $\mathcal{M}=\{\ell \in \mathbb{L} \mid \operatorname{Var}(\{\ell\}) \cap \operatorname{Var}(\Sigma)=\emptyset\}$ such that there does not exist a clause $C \in \mathcal{S}$ with $|\operatorname{Var}(C) \backslash \operatorname{Var}(\Sigma)|=1$. In other words, the decisions $\mathcal{M}$ do not lead to logical follow-up assignments, i.e., implications. The reason for this restriction is that implications may lead to conflicts (clauses that cannot be satisfied). When a single decision is made, this decision needs to be rolled back when a conflict is caused, but when multiple 


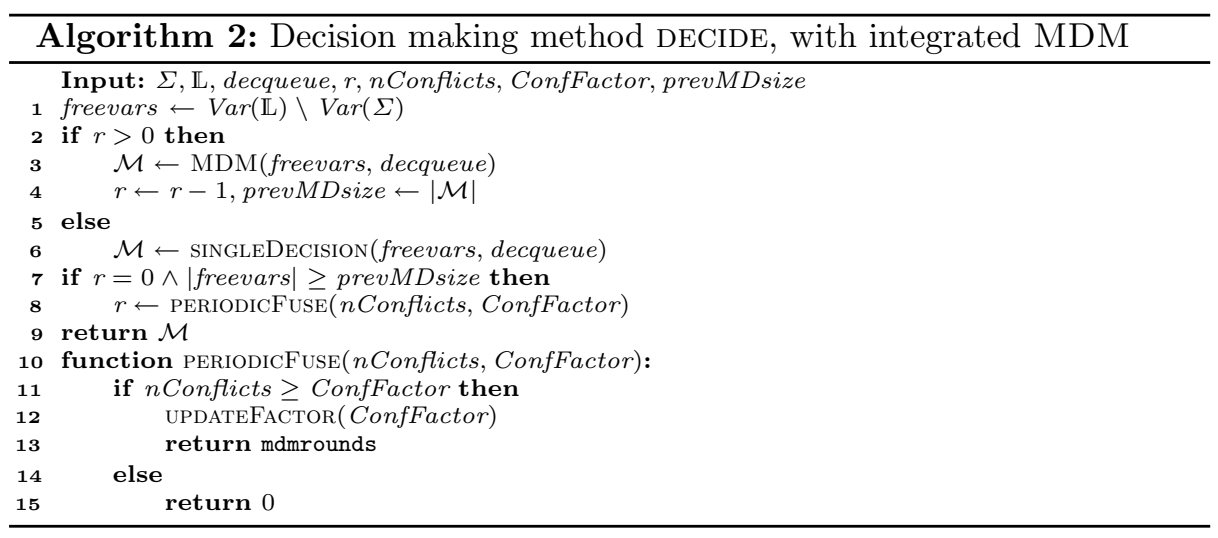

decisions are made, detecting which decisions actually cause a conflict is more difficult. Note that MDM cannot always make multiple decisions; implications are needed to solve a formula, so single decisions still have to be made frequently.

In [30], MDM was integrated into MiniSAT and GLuCose, and since multiple decisions should be selected periodically, a mechanism was proposed that decides when to make multiple decisions based on the solver restart policy. However, since solvers can differ greatly in this policy, we wanted to create an alternative mechanism not depending on this. PARAFROST is based on CADiCAL [6], which has a very different restart policy compared to MiniSat and Glucose.

Algorithm 2 presents PARAFROST's DECIDE method, which is called every time a decision must be made. Besides $\Sigma$ and $\mathbb{L}$, it is given a queue decqueue, in which the variables are ordered based on a decision heuristic. In PARAFROST, the heuristics Variable State Independent Decaying Sum (VSIDS) [27] and Variable Move-To-Front (VMTF) [7] are alternatingly used. The latter was not used before in [30]. DECIDE also gets a variable $r$, initially set to the constant mdmrounds. These values are used to control the periodic call of MDM, in which a set of multiple decisions is made per round. Experiments have shown that mdmrounds $=3$ is effective [30]. Finally, the number of conflicts so far $(n C o n-$ flicts), a variable ConfFactor used to switch MDM on and off, and a variable prevMDsize, storing the size of the most recent set of multiple decisions, are given.

To select new decisions, the set of unassigned variables is created at line 1. If we are calling MDM mdmrounds times (line 2), then MDM is called again and $r$ is updated. The alternative is to make a single decision (line 6). If we have stopped calling MDM, and enough unassigned variables are present (line 7), method PERIODICFUSE is called, which either sets $r$ back to mdmrounds or to 0 , depending on $n$ Conflicts (lines 10-15). There are enough unassigned variables if there are more unassigned variables than variables in the most recent multiple decisions set. In PERIODICFUSE, nConflicts is compared to ConfFactor, which is initially set to a configurable value (default 2,000). ConfFactor is updated using 
a function UpdateFACTOR. This makes ConfFactor grow linearly, to achieve a suitable balance between ConfFactor and $n$ Conflicts as the solving progresses.

\section{Benchmarks}

We conducted experiments with CBMC in combination with MiniSAT (the default), Glucose, CaDiCaL, Parafrost, Parafrost with MDM, and a CPU-only version, referred to as PARAFROST (NOGPU). ${ }^{4}$ We used the AWS benchmarks in which the data structures hash table, array list, array buff, linked list, priority queue, byte cursor and string were analysed. The loop unwinding upper-bounds 8, 16, 64, 128 and 1,000 were used, resulting in 168 different verification problems.

All experiments were executed on the DAS-5 cluster [4]. Each program was verified in isolation on a separate node, with a time-out of 3,600 s. Each node had an Intel Xeon E5-2630 CPU $(2.4 \mathrm{GHz})$ with $64 \mathrm{~GB}$ of memory, and an NVIDIA RTX $2080 \mathrm{Ti}$, with $68 \mathrm{SMs}$ (64 cores/SM) and $11 \mathrm{~GB}$ global memory.

Figure 4 presents the decision procedure runtime, and how much time was spent on VCE. PARAFROST outperforms all sequential solvers including CADICAL (plot 4a). Even though PARAFROST is based on CADiCAL, its different data structures, simplification mechanism and parameters tuned for large formulas makes PARAFROST more effective in these experiments. MDM further improves PARAFROST. Plot 4b demonstrates that CBMC with MiniSAT often spends most of the time on VCE. PARAFROST significantly reduces the time spent on VCE compared to other solvers.

In Table 1, the Verified column lists per solver the number of verified programs, and PAR-2 gives the penalized average runtime-2 metric. PAR-2 score accumulates the running times of all solved instances with $2 \times$ the time-out of unsolved ones, divided by the total number of formulas. The solver with the lowest score is the winner. The triangles $\boldsymbol{\Delta}$ and $\boldsymbol{\nabla}$ mean significantly better and worse, respectively. The MiniSAT column lists how many programs were verified faster with the other solvers compared to MiniSAT. Between parentheses, it is given how many of those programs were not solved by MiniSAT at all. The final four columns serve the same purpose for the other solvers. For example, PARAFROST-MDM verified 123 programs faster than CADICAL, in which 12 could not be verified by the latter. The last two rows provide a similar comparison. Clearly, PARAFROST-MDM verified the largest number of programs, with the lowest score.

Figure 5 presents the speedups of the PARAFROST configurations for the individual cases. Overall, SAT solving was accelerated effectively with PARAFROST and PAraFrost-MDM. Compared to ParaFrost (NOGPU), PARAFROST (and PARAFROST-MDM), accelerated multiple instances by up to $18 \times$ (and $27 \times$ ), and the geometric average speedup for all programs was $1.3 \times($ and $1.6 \times)$.

\footnotetext{
${ }^{4}$ We also tried to use CBMC with Z3, but were not able to correctly configure this combination at the time of writing.
} 




(a) Verification time (timeout: 3600 seconds)

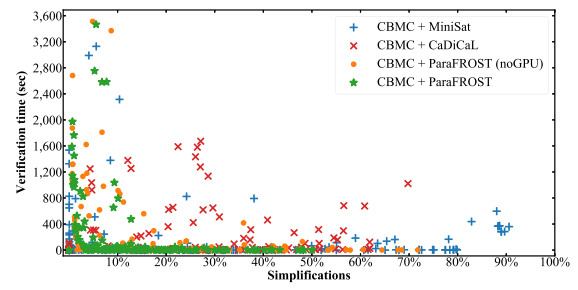

(b) Percentage of verification time used for VCE

Fig. 4. CBMC runtimes for all solvers over the benchmark suite.

Table 1. CBMC performance analysis using the various solvers.

\begin{tabular}{|c|c|c|c|c|c|c|c|c|c|c|}
\hline Configuration & Verified & PAR-2 & & iniSat & & Glucose & & CaDiCaL & PFCPU & PFGPU \\
\hline $\mathrm{CBMC}+$ MiniSat & 143 & 1219 & & $\mathrm{n} / \mathrm{a}$ & & $\mathrm{n} / \mathrm{a}$ & & $\mathrm{n} / \mathrm{a}$ & $\mathrm{n} / \mathrm{a}$ & $\mathrm{n} / \mathrm{a}$ \\
\hline CBMC + Glucose & 139 & V 1388 & ק 49 & $(-4)$ & & $\mathrm{n} / \mathrm{a}$ & & $\mathrm{n} / \mathrm{a}$ & $\mathrm{n} / \mathrm{a}$ & $\mathrm{n} / \mathrm{a}$ \\
\hline $\mathrm{CBMC}+\mathrm{CaDiCaL}$ & 143 & 1226 & & 43 & & $53 \quad(+4)$ & & $\mathrm{n} / \mathrm{a}$ & $\mathrm{n} / \mathrm{a}$ & $\mathrm{n} / \mathrm{a}$ \\
\hline $\mathrm{CBMC}+\mathrm{PFCPU}$ & 154 & 824 & 5 & $(+11)$ & & $62(+15)$ & & $83(+11)$ & $\mathrm{n} / \mathrm{a}$ & $\mathrm{n} / \mathrm{a}$ \\
\hline $\mathrm{CBMC}+\mathrm{PFGPU}$ & 155 & $\triangle 765$ & $\Delta 66$ & $(+12)$ & $\Delta$ & $83(+16)$ & $\Delta$ & $96(+12)$ & $120(+1)$ & $\mathrm{n} / \mathrm{a}$ \\
\hline CBMC + PFGPU-MDM & 155 & $\triangle 743$ & $\Delta 84$ & $(+12)$ & $\Delta$ & $102(+16)$ & $\Delta$ & $123(+12)$ & $133(+1)$ & 121 \\
\hline
\end{tabular}

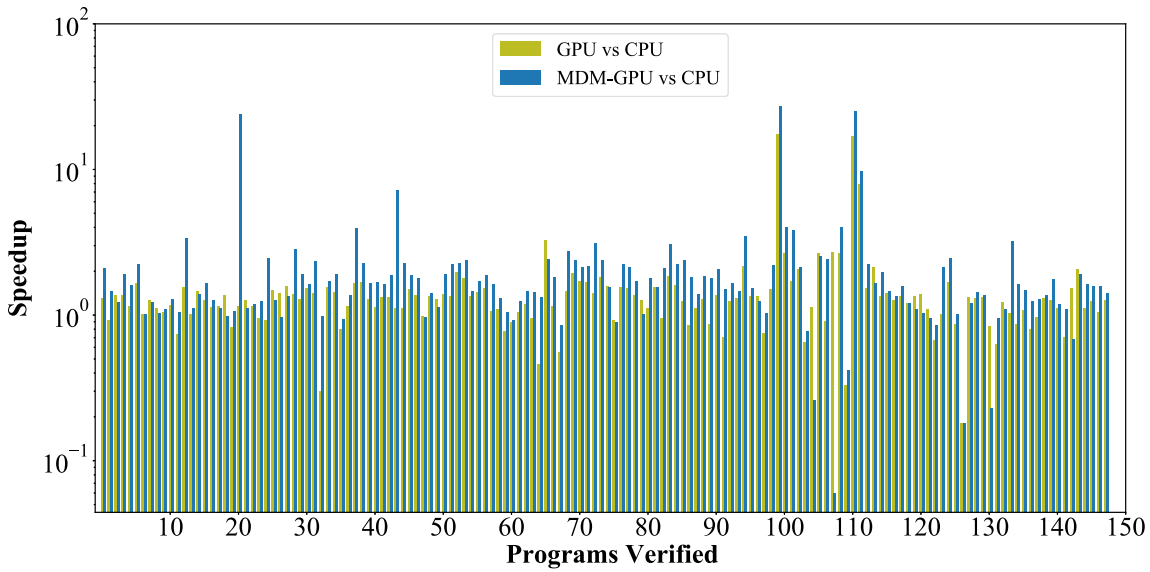

Fig. 5. Speedups of the individual cases.

\section{Conclusion}

We have presented PARAFROST, the first tool to accelerate BMC using GPUs. Given that BMC formulas tend to have much redundancy, PARAFROST effectively reduces solving times with GPU pre- and inprocessing, and by using MDM, which is particularly effective when many variables are present. In the future, we will combine our approach with (existing) multi-threaded BMC. We expect these techniques to strengthen each other. 


\section{References}

1. Ábrahám, E., Schubert, T., Becker, B., Fränzle, M., Herde, C.: Parallel SAT solving in bounded model checking. J. Logic Comput. 21(1), 5-21 (2009)

2. Amazon: The Amazon Web Services Core C99 Package Benchmark Set (2021). https://github.com/awslabs/aws-c-common/tree/main/verification/cbmc/proofs

3. Audemard, G., Simon, L.: Predicting Learnt Clauses Quality in Modern SAT Solvers. In: IJCAI, pp. 399-404. Morgan Kaufmann Publishers Inc. (2009)

4. Bal, H., et al.: A medium-scale distributed system for computer science research: infrastructure for the long term. IEEE Comput. 49(5), 54-63 (2016)

5. Biere, A., Cimatti, A., Clarke, E., Zhu, Y.: Symbolic model checking without BDDs. In: Cleaveland, W.R. (ed.) TACAS 1999. LNCS, vol. 1579, pp. 193-207. Springer, Heidelberg (1999). https://doi.org/10.1007/3-540-49059-0_14

6. Biere, A., Fazekas, K., Fleury, M., Heisinger, M.: CaDiCaL, Kissat, Paracooba, Plingeling and Treengeling entering the SAT Competition 2020. In: SAT Competition 2020, pp. 51-53 (2020)

7. Biere, A., Fröhlich, A.: Evaluating CDCL variable scoring schemes. In: Heule, M., Weaver, S. (eds.) SAT 2015. LNCS, vol. 9340, pp. 405-422. Springer, Cham (2015). https://doi.org/10.1007/978-3-319-24318-4_29

8. Bošnački, D., Edelkamp, S., Sulewski, D., Wijs, A.: GPU-PRISM: an extension of PRISM for general purpose graphics processing units. In: PDMC-HiBi, pp. 17-19. IEEE Computer Society (2010)

9. Bošnački, D., Odenbrett, M., Wijs, A., Ligtenberg, W., Hilbers, P.: Efficient reconstruction of biological networks via transitive reduction on general purpose graphics processors. BMC Bioinform. 13(281) (2012)

10. Bradley, A.R.: SAT-based model checking without unrolling. In: Jhala, R., Schmidt, D. (eds.) VMCAI 2011. LNCS, vol. 6538, pp. 70-87. Springer, Heidelberg (2011). https://doi.org/10.1007/978-3-642-18275-4_7

11. Brown, C.E.: Reducing higher-order theorem proving to a sequence of SAT problems. J. Autom. Reason. 51(1), 57-77 (2013)

12. Burch, J.R., Clarke, E.M., McMillan, K.L., Dill, D.L.: Sequential circuit verification using symbolic model checking. In: DAC, pp. 46-51. IEEE (1990)

13. Chatterjee, P., Roy, S., Diep, B., Lal, A.: Distributed bounded model checking. In: FMCAD, pp. 47-56. TU Wien Academic Press (2020)

14. Clarke, E., Kroening, D., Lerda, F.: A tool for checking ANSI-C programs. In: Jensen, K., Podelski, A. (eds.) TACAS 2004. LNCS, vol. 2988, pp. 168-176. Springer, Heidelberg (2004). https://doi.org/10.1007/978-3-540-24730-2_15

15. Eén, N., Biere, A.: Effective preprocessing in SAT through variable and clause elimination. In: Bacchus, F., Walsh, T. (eds.) SAT 2005. LNCS, vol. 3569, pp. 61-75. Springer, Heidelberg (2005). https://doi.org/10.1007/11499107_5

16. Eén, N., Sörensson, N.: Temporal induction by incremental SAT solving. Electron. Notes Theor. Comput. Sci. 89(4), 543-560 (2003)

17. Eén, N., Sörensson, N.: An extensible SAT-solver. In: Giunchiglia, E., Tacchella, A. (eds.) SAT 2003. LNCS, vol. 2919, pp. 502-518. Springer, Heidelberg (2004). https://doi.org/10.1007/978-3-540-24605-3_37

18. Hamadi, Y., Jabbour, S., Sais, L.: ManySAT: a parallel SAT solver. J. Satisf. 6, 245-262 (2009)

19. Inverso, O., Trubiani, C.: Parallel and distributed bounded model checking of multi-threaded programs. In: PPoPP, pp. 202-216. ACM (2020) 
20. Järvisalo, M., Heule, M.J.H., Biere, A.: Inprocessing rules. In: Gramlich, B., Miller, D., Sattler, U. (eds.) IJCAR 2012. LNCS (LNAI), vol. 7364, pp. 355-370. Springer, Heidelberg (2012). https://doi.org/10.1007/978-3-642-31365-3_28

21. Kahsai, T., Tinelli, C.: PKind: a parallel k-induction based model checker. In: PDMC, EPTCS, vol. 72, pp. 55-62. Open Publishing Association (2011)

22. Kroening, D., Strichman, O.: Quantified formulas. In: Decision Procedures. TTCSAES, pp. 199-227. Springer, Heidelberg (2016). https://doi.org/10.1007/978-3662-50497-0_9

23. Leiserson, C.E., et al.: There's plenty of room at the top: what will drive computer performance after Moore's law? Science 368(6495) (2020)

24. Lynce, I., Marques-Silva, J.: Probing-based preprocessing techniques for propositional satisfiability. In: ICTAI, pp. 105-110. IEEE (2003)

25. Marques-Silva, J., Glass, T.: Combinational equivalence checking using satisfiability and recursive learning. In: DATE, pp. 145-149. ACM, March 1999

26. Marques-Silva, J.P., Sakallah, K.A.: GRASP: a search algorithm for propositional satisfiability. IEEE Trans. Comput. 48(5), 506-521 (1999)

27. Moskewicz, M.W., Madigan, C.F., Zhao, Y., Zhang, L., Malik, S.: Chaff: engineering an efficient SAT solver. In: DAC, pp. 530-535. ACM (2001)

28. NVIDIA: CUDA C Programming Guide (2020). https://docs.nvidia.com/cuda/ cuda-c-programming-guide

29. Oostema, P., Martins, R., Heule, M.: Coloring unit-distance strips using SAT. In: LPAR23. EPiC Series in Computing, vol. 73, pp. 373-389. EasyChair (2020)

30. Osama, M., Wijs, A.: Multiple decision making in conflict-driven clause learning. In: ICTAI, pp. 161-169. IEEE (2020)

31. Osama, M., Gaber, L., Hussein, A.I., Mahmoud, H.: An efficient SAT-based test generation algorithm with GPU accelerator. J. Electron. Test. 34(5), 511-527 (2018). https://doi.org/10.1007/s10836-018-5747-4

32. Osama, M., Wijs, A.: Parallel SAT simplification on GPU architectures. In: Vojnar, T., Zhang, L. (eds.) TACAS 2019. LNCS, vol. 11427, pp. 21-40. Springer, Cham (2019). https://doi.org/10.1007/978-3-030-17462-0_2

33. Osama, M., Wijs, A.: SIGmA: GPU accelerated simplification of SAT formulas. In: Ahrendt, W., Tapia Tarifa, S.L. (eds.) IFM 2019. LNCS, vol. 11918, pp. 514-522. Springer, Cham (2019). https://doi.org/10.1007/978-3-030-34968-4_29

34. Osama, M., Wijs, A., Biere, A.: SAT solving with GPU accelerated inprocessing. In: TACAS 2021. LNCS, vol. 12651, pp. 133-151. Springer, Cham (2021). https:// doi.org/10.1007/978-3-030-72016-2_8

35. Phan, Q.S., Malacaria, P., Pasareanu, C.: Concurrent bounded model checking. ACM SIGSOFT Softw. Eng. Notes 40(1), 1-5 (2015)

36. Piette, C., Hamadi, Y., Saïs, L.: Vivifying propositional clausal formulae. In: ECAI, pp. 525-529. IOS Press, NLD (2008)

37. Sengupta, S., Harris, M., Garland, M., Owens, J.: Efficient parallel scan algorithms for manycore GPUs. In: SCMA, pp. 413-442. Taylor \& Francis (2011)

38. Sheeran, M., Singh, S., Stålmarck, G.: Checking safety properties using induction and a SAT-solver. In: Hunt, W.A., Johnson, S.D. (eds.) FMCAD 2000. LNCS, vol. 1954, pp. 127-144. Springer, Heidelberg (2000). https://doi.org/10.1007/3540-40922-X_8

39. Shtrichman, O.: Pruning techniques for the SAT-based bounded model checking problem. In: Margaria, T., Melham, T. (eds.) CHARME 2001. LNCS, vol. 2144, pp. 58-70. Springer, Heidelberg (2001). https://doi.org/10.1007/3-540-44798-9_4 
40. Wijs, A.: BFS-based model checking of linear-time properties with an application on GPUs. In: Chaudhuri, S., Farzan, A. (eds.) CAV 2016. LNCS, vol. 9780, pp. 472-493. Springer, Cham (2016). https://doi.org/10.1007/978-3-319-41540-6_26

41. Wijs, A., Neele, T., Bošnački, D.: GPUexplore 2.0: unleashing GPU explicit-state model checking. In: Fitzgerald, J., Heitmeyer, C., Gnesi, S., Philippou, A. (eds.) FM 2016. LNCS, vol. 9995, pp. 694-701. Springer, Cham (2016). https://doi.org/ 10.1007/978-3-319-48989-6_42

42. Youness, H., Ibraheim, A., Moness, M., Osama, M.: An efficient implementation of ant colony optimization on GPU for the satisfiability problem. In: PDP, pp. 230-235 (2015)

Open Access This chapter is licensed under the terms of the Creative Commons Attribution 4.0 International License (http://creativecommons.org/licenses/by/4.0/), which permits use, sharing, adaptation, distribution and reproduction in any medium or format, as long as you give appropriate credit to the original author(s) and the source, provide a link to the Creative Commons license and indicate if changes were made.

The images or other third party material in this chapter are included in the chapter's Creative Commons license, unless indicated otherwise in a credit line to the material. If material is not included in the chapter's Creative Commons license and your intended use is not permitted by statutory regulation or exceeds the permitted use, you will need to obtain permission directly from the copyright holder.

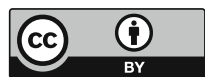

